\title{
Newly Described Clinical and Immunopathological Feature of Dermatitis Herpetiformis
}

\author{
Veronica Bonciolini, ${ }^{1}$ Diletta Bonciani, ${ }^{1}$ Alice Verdelli, ${ }^{1}$ Antonietta D'Errico, ${ }^{1}$ \\ Emiliano Antiga, ${ }^{1,2}$ Paolo Fabbri, ${ }^{1}$ and Marzia Caproni ${ }^{1}$ \\ ${ }^{1}$ Department of Critical Care, Section of Dermatology, University of Florence, Piazza Indipendenza, 11, 50129 Florence, Italy \\ ${ }^{2}$ Department of Clinical Physiopathology, University of Florence, 50139 Florence, Italy
}

Correspondence should be addressed to Veronica Bonciolini, vbonciolini@gmail.com

Received 13 February 2012; Accepted 13 April 2012

Academic Editor: Alessio Fasano

Copyright ( 2012 Veronica Bonciolini et al. This is an open access article distributed under the Creative Commons Attribution License, which permits unrestricted use, distribution, and reproduction in any medium, provided the original work is properly cited.

\begin{abstract}
Dermatitis herpetiformis $(\mathrm{DH})$ is an inflammatory cutaneous disease with typical histopathological and immunopathological findings clinically characterized by intensely pruritic polymorphic lesions with a chronic-relapsing course. In addition to classic clinical manifestations of $\mathrm{DH}$, atypical variants are more and more frequently reported and histological and immunological are added to them, whereas the impact on quality of life of patients with $\mathrm{DH}$ is increasingly important to a certain diagnosis. The aim of this paper is to describe all the possible clinical, histological, and immunological variants of $\mathrm{DH}$ in order to facilitate the diagnosis of a rare disease and, therefore, little known.
\end{abstract}

\section{Introduction}

Dermatitis herpetiformis (DH) is an inflammatory cutaneous disease with typical histopathological and immunopathological findings clinically characterized by intensely pruritic polymorphic lesions with a chronic-relapsing course, first described by Duhring in 1884 [1]. In 1966, Marks et al. [2]. reported small-bowel changes in patients with $\mathrm{DH}$ and later gastrointestinal abnormalities described in patients affected by $\mathrm{DH}$ were found to be the same as in those with celiac disease (CD) [3]. Currently, DH is considered as the cutaneous manifestation of gluten-dependent enteropathy, corresponding to CD.

$\mathrm{DH}$ is an autoimmune disease, a finding that is strongly supported by landmark studies revealing the granular deposition of immunoglobulin A (IgA) in the skin [4-6]. The same type of immunoglobulin was detected in the small intestinal mucosa of patient affected by $\mathrm{CD}$, even before the development of the gluten-induced flat jejunal lesions. This observation on one hand emphasized the pivotal pathogenetic role of these immune deposits and from the other side represented a link between the two diseases $[7,8]$.

\section{Clinical Features}

The clinical morphology, in particular a polymorphous presentation and distribution of the lesions, are the hallmarks of the DH. Primary lesions of DH consist of grouped erythematous papules, urticarial plaques surmounted by vesicles or also blisters, which may be often replaced by erosions and excoriations, because of the intense itching characteristically associated with this condition. Chronic pruritus and excoriations might lead to lichenification, furthermore a transient postinflammatory hyperpigmentation may occur when the lesion resolve [9-13]. The symmetrical distribution of the herpetiform lesions on the extensor surfaces of the elbows (90\%), knees (30\%), shoulders, middle line of the back, buttocks, and sacral region is also a typical feature of the disease. In particular, Cottini [14]. In 1955 first described a clinical variant with exclusive localization of the lesions on the knees and elbows symmetrically. Anyway, scalp, nuchal area, face, and groin may be also involved. No clinical differences were described between darker- and white-skinned individuals, although DH remains primarily a prerogative of Caucasian population [15]. Most of the patients suffer 
not only itching but also tickle or burning sensation even before the onset of the skin lesions.

An uncommon skin manifestation of $\mathrm{DH}$ is represented by purpuric lesions occasionally described on palmo-plantar surfaces of children, but rarely reported in adults. First descriptions of $\mathrm{DH}$ presenting as purpuric, erythematous, or hemorrhagic palmar or plantar lesions date from 1971 $[16,17]$. Fraser et al. reported such lesions in four of 14 patients with DH [17]. In 1979, Katz and Marks noted that vesicles may be hemorrhagic, particularly if they are located on the hands [18]. Moulin et al. [19] eventually described reported four DH patients with palmar "pseudopurpuric lesions" which showed typical histologic changes of $\mathrm{DH}$ in three out of four cases. Pierce et al. [20] described a 27-year-old man with typical DH and additional purpuric lesions on the palms. In 1986, 47 pediatric series of $47 \mathrm{DH}$ cases by Karpati et al. [21], 30 (64\%) showed red-brown palmar purpuric lesions, which, however, were not biopsied. Sometimes, petechial lesions on the fingertips may be the only symptom of DH, as reported by Moulin et al. [19], Rutten and Goos [22], Hofmann et al. [23], and recently Flann et al. [24]. Finally, the last case was described in 2011 by Heinlin et al. [25]. In a 15-year-old female with a 6-month history of recurrent painful petechiae on the fingers and feet, that was diagnosed as DH after histopathology, direct- and indirect- immunofluorescence (DIF, IIF).

However, atypical clinical presentation of $\mathrm{DH}$ reported in the literature includes also palmoplantar keratosis, wheals of chronic urticaria and lesions mimicking prurigo pigmentosa [26-28]. In particular, Ohshima et al. [26] in 2003 described a 63-year-old Japanese man which presented palmoplantar keratosis, in addition to itchy areas of erythema on the buttocks and knees with small blisters on the border that were clinically, histologically, and immunologically compatible with DH. The histologic evaluation of palmoplantar keratotic lesions showed hyperkeratosis, acanthosis, and cellular infiltration, mainly of lymphocytes, in the dermal papillae, aspects that were more compatible with psoriasis, but DIF showed granular IgA deposits in the dermal papillae confirming the diagnosis of $\mathrm{DH}$. An unusual clinical presentation of DH in children described instead by Powell et al. [27] in 2004 consisted of chronic urticaria-like skin lesions. Histologic evaluation showed neutrophilic microabscesses in the dermal papilla with subepidermal blister formation, suggestive of $\mathrm{DH}$, that was confirmed by fibrillar IgA deposition along the basement membrane zone (BMZ) with papillary accentuation revealed by DIF. Finally, Saito et al. [28] in 2005 described another atypical clinical presentation of $\mathrm{DH}$, that was also in a Japanese subject, with features of prurigo pigmentosa but characterized by both histological and immunological aspect of $\mathrm{DH}$.

\section{Histopathology}

The classic histopathologic features of $\mathrm{DH}$ seen on light microscopy include a subepidermal cleft with neutrophils, that are considered the most likely responsible for the dermal-epidermal separation [29], and a few eosinophils at the tips of dermal papillae, that are often accompanied by a perivascular mixed inflammatory infiltrate $[12,26]$. While these findings are characteristic, there are a number of patients who present with pruritic, excoriated skin lesions with clinical and immunological features of $\mathrm{DH}$ in whom the histologic findings are nonspecific and do not confirm the diagnosis as showed by Warren and Cockerell [30], which found that $37.5 \%$ of $\mathrm{DH}$ patients had hematoxylin and eosin findings of a lymphocytic infiltrate only with fibrosis in the dermal papillae and ectatic capillaries. The authors hypothesized that the nonspecific histologic findings could represent both a sampling error on the part of the clinician taking the biopsy, in particular, choosing excoriated lesions that correspond to a later stage of the disease, or the pathology laboratory in sampling the lesion for histology, considering appropriate a progressive cutting of the tissue block, or distinct subgroup of dermatitis herpetiformis, possibly with a separate antigenic target [30].

\section{DIF}

Actually DIF of uninvolved skin collected in the perilesional site is considered as the diagnostic gold standard for $\mathrm{DH}$ [12]. The choice of normal appearing perilesional skin as site of biopsy specimen for DIF is not random, because in this site $\mathrm{DH}$ patients showed greater IgA deposition than in nonlesional or lesional skin as demonstrated by Zone et al. [4].

Two different patterns are possible: (a) granular deposits in the dermal papillae and (b) granular deposits along the basement membrane. In both cases, deposits are thought to be polyclonal but are mainly composed of IgA1 [31]. The two patterns may also be present as a combination resulting in granular IgA deposition along the basement membrane with accentuation at the tips of the dermal papillae [9-11, 32]. A third different pattern, presents in $50 \%$ of Japanese populations, first described in 1993 by Kawana and Segawa [33] as "fibrillar pattern," was subsequently reconsidered by Ko et al. [34] in 2010. The fundamental difference between this last and previous is that the IgA deposition presents as linear streaks rather than fine granules in the papillary dermis. As suggested by Ko et al., the fibrillar pattern of IgA deposition may correlate with a clinical variant of $\mathrm{DH}$ or another as yet undefined disorder, and some case reports of atypical clinical presentations, that may be urticarial or psoriasiform, support this hypothesis $[35,36]$. The correlation between the fibrillar DIF pattern and DH is, however, still debated. Although often the granular and the fibrillar patterns are associated in patients with $\mathrm{DH}$, the last one alone more often correlates with atypical features, including atypical clinical presentations, absence of HLA-B8/DR3DQ2 haplotype, and lack of gluten-sensitive enteropathy or detectable circulating autoantibodies [37]. However, not all authors agree that, considering that the two different pattern may be the expression of a different method of sectioning [33].

\section{Serologic Findings}

Serologic testing is a useful adjunct to tissue-based studies. Contrary to the other bullous disorders, DH patients have no circulating autoantibodies binding to the cutaneous 
basement membrane components or to other adherent structures of the skin, but they have gluten-induced IgA autoantibodies against transglutaminase (TG) 2 and TG3 also called tissue-TG (t-TG) and epidermal-TG (e-TG), respectively [13].

TG represents an evolutionary conserved family of $\mathrm{Ca}^{+2}$. dependent enzymes that covalently cross-link or modify proteins by formation of an isopeptide bond between a peptidebound glutamine residue and a primary amine, most commonly a lysine residue either within the same or a neighboring polypeptide chain [38]. Nine human types of TG were identified and some of them are expressed in the epidermis. TG2, among them, is the best characterized and also the most abundant and widely distributed [39]. Over time, different authors demonstrated that the enzymatic activity of TG2 is implicated in several diseases as Huntington disease, Alzheimer disease, and CD [40-42]. In particular, CD TG2 catalyzes highly specific deamidation of gluten peptides improving the binding of the peptides to the diseaseassociated HLA DQ2 and DQ8 molecules and becoming essential for the T-cell-mediated immune response against gluten. TG2 is also the primary autoantigen of CD recognized by autoantibodies of IgA1 class. These autoantibodies are considered the main serological marker of CD as diseasespecific and more generally of gluten-sensitivity diseases and therefore of DH $[12,38,43]$. Levels of anti-TG2 correlate with bowel damage and gluten-free diet adherence in $\mathrm{DH} / \mathrm{CD}$ patients and are measured using an enzyme-linked immunosorbent assay (ELISA) [44]. In DH, some authors have demonstrated an IgA anti-tTG specificity higher than $90 \%$, and a sensitivity ranging from $47 \%$ to $95 \%$ [45-49]. However, as demonstrated by Sárdy et al. [50] the target autoantigen of $\mathrm{DH}$ is represented by TG3, that shares a $64 \%$ of homology with TG2 and overlapping sensitivity and specificity. As recently demonstrated by Stamnaes et al. [39] is also able to accommodate gluten peptides as substrates and form either thioester or isopeptide-linked complexes with these peptides stimulating the immune system's response, even if it is still unclear how it gets to the skin lesions. Although promising, the anti-eTG assay has not yet been approved in the United States for in vitro use to diagnose $\mathrm{DH}$ [51].

In addition to anti-TG2 antibodies, also those anti-EMA have become relatively sensitive and specific tools for initial detection of gluten-sensitive disease and, therefore, of DH. In particular, anti-EMA antibodies belong to the IgA1 subclass and are directed against primate smooth muscle reticular connective tissue. The detection of EMA is based on an indirect immunofluorescence assay on monkey oesophagus and it is more time-consuming and operator-dependent than the one of anti-TG2 ELISA testing [52], showing a specificity close to $100 \%$, and a sensitivity ranging from $52 \%$ to $100 \%$ for the diagnosis of $\mathrm{DH}[44-47,53]$.

Other autoantibodies are shared between DH and CD such as antigliadin antibodies and antireticulin antibodies, without, however, the same diagnostic value of anti-TG2 and anti-EMA.

In 2006, Sugai et al. first showed that the most reliable diagnostic test to identify gluten sensitivity in $\mathrm{DH}$ patients was the detection of antibodies against deamidated synthetic gliadin-derived peptides, both IgA and IgG isotypes [54]. Recently, a new CD serum marker based on TG2 covalently cross-linked to deamidated gliadin peptides, coined "neoepitope," was carefully studied by Matthias et al. and data supporting the use of this new assay (AESKULISA CeliCheck New Generation) in CD diagnosis were obtained in a longitudinal study of 2684 eligible subjects demonstrating a sensitivity of $92,31 \%$ and a specificity of $82,89 \%$, that were highest when compared to TG2 ELISA test or antiEMA tests [55]. The diagnostic value of these antibodies was also confirmed by Jaskowski et al. [56], which demonstrated the superior sensitivity of anti-tTG/DGP EIA screen (IgA + IgG) compared to IgA anti-tTG both in pediatric CD $(92,6 \%$ versus $90,7 \%$ ) and adult $\mathrm{DH}$ (65\% versus $48,8 \%$ and $62 \%$ versus $44 \%$ in retrospective and prospective sera, resp.). Furthermore, very recently, Kasperkiewicz et al. [57] showed the higher sensitivity of the anti-GAF3X ELISA, a novel CD serologic assay using deamidated gliadin-analogous fusion peptides, to detect CD-associated autoantibodies in patients with $\mathrm{DH}$ compared with tests using native gliadin, tTG or endomysium as substrates.

\section{Conclusion}

From the data reported above, it seems evident that the diagnosis of $\mathrm{DH}$ can be difficult and, therefore, requires a complex approach, that should be clinical, histological and immunological, having regards to the atypical variants more and more frequently described in the literature. This becomes crucial since, as it is known, patients with $\mathrm{DH}$ present a significant reduction in their quality of life, mainly due to the need of a lifelong gluten-free diet and, consequently, to a significant change in lifestyle and eating habits.

\section{Acknowledgment}

This work was supported by the Ministry of Instruction, University and Research of the Italian Government (PRIN 2008EW3FHK).

\section{References}

[1] L. A. Duhring, "Dermatitis herpetiformis," Journal of the American Medical Association, vol. 250, no. 2, pp. 212-216, 1983.

[2] J. Marks, S. Shuster, and A. J. Watson, "Small-bowel changes in dermatitis herpetiformis," The Lancet, vol. 2, no. 7476, pp. 1280-1282, 1966.

[3] L. Fry, P. Keir, R. M. McMinn, J. D. Cowan, and A. V. Hoffbrand, "Small-intestinal structure and function and haematological changes in dermatitis herpetiformis," The Lancet, vol. 2, no. 7519, pp. 729-733, 1967.

[4] J. J. Zone, L. J. Meyer, and M. J. Petersen, "Deposition of granular IgA relative to clinical lesions in dermatitis herpetiformis," Archives of Dermatology, vol. 132, no. 8, pp. 912-918, 1996.

[5] L. Fry, "Dermatitis herpetiformis: problems, progress and prospects," European Journal of Dermatology, vol. 12, no. 6, pp. 523-531, 2002. 
[6] L. Fry and P. P. Seah, "Dermatitis herpetiformis: an evaluation of diagnostic criteria," British Journal of Dermatology, vol. 90, no. 2, pp. 137-146, 1974.

[7] S. Kárpáti, "Dermatitis herpetiformis: close to unravelling a disease," Journal of Dermatological Science, vol. 34, no. 2, pp. 83-90, 2004.

[8] J. J. Zone, "Skin manifestations of celiac disease," Gastroenterology, vol. 128, no. 4, pp. S87-S91, 2005.

[9] L. Fry, "Dermatitis herpetiformis: problems, progress and prospects," European Journal of Dermatology, vol. 12, no. 6, pp. 523-531, 2002.

[10] S. W. Yeh, B. Ahmed, N. Sami, and A. R. Ahmed, "Blistering disorders: diagnosis and treatment," Dermatologic Therapy, vol. 16, no. 3, pp. 214-223, 2003.

[11] M. E. O. Nicolas, P. K. Krause, L. E. Gibson, and J. A. Murray, "Dermatitis herpetiformis," International Journal of Dermatology, vol. 42, no. 8, pp. 588-600, 2003.

[12] M. Caproni, E. Antiga, L. Melani, and P. Fabbri, "Guidelines for the diagnosis and treatment of dermatitis herpetiformis," Journal of the European Academy of Dermatology and Venereology, vol. 23, no. 6, pp. 633-638, 2009.

[13] S. Kàrpàti, "Dermatitis herpetiformis," Clinics in Dermatology, vol. 30, pp. 56-59, 2012.

[14] G. B. Cottini, "Symmetrical dermatitis herpetiformis of Duhring localized on the knees and elbows," Annales de Dermatologie et de Syphiligraphie, vol. 82, pp. 285-286, 1955.

[15] D. Bolotin and V. Petronic-Rosic, "Dermatitis herpetiformis: part I. Epidemiology, pathogenesis, and clinical presentation," Journal of the American Academy of Dermatology, vol. 64, no. 6, pp. 1017-1024, 2011.

[16] R. Marks and E. W. Jones, "Purpura in dermatitis herpetiformis," British Journal of Dermatology, vol. 84, no. 4, pp. 386$388,1971$.

[17] N. G. Fraser, J. S. Beck, and F. Albert-Recht, "Serum complement (C'3) and immunoglobulin levels in dermatitis herpetiformis," British Journal of Dermatology, vol. 85, no. 4, pp. 314-319, 1971.

[18] S. I. Katz and J. M. Marks, "Dermatitis herpetiformis," in Dermatology in General Medicine, T. B. Fitzpatrick, Ed., pp. 326-330, McGraw-Hill, New York, NY, USA, 2nd edition, 1979.

[19] G. Moulin, D. Barrut, and M. P. Franc, "Pseudopurpuric palmar localizations of herpetiform dermatitis," Annales de Dermatologie et de Venereologie, vol. 110, no. 2, pp. 121-126, 1983.

[20] D. K. Pierce, S. M. Purcell, and R. L. Spielvogel, "Purpuric papules and vesicles of the palms in dermatitis herpetiformis.," Journal of the American Academy of Dermatology, vol. 16, no. 6, pp. 1274-1276, 1987.

[21] S. Karpati, E. Torok, and I. Kosnai, "Discrete palmar and plantar symptoms in children with dermatitis herpetiformis Duhring," Cutis, vol. 37, no. 3, pp. 184-187, 1986.

[22] A. Rutten and M. Goos, "Palmoplantar purpura in dermatitis herpetiformis Duhring," Hautarzt, vol. 40, no. 10, pp. 640 $643,1989$.

[23] S. C. Hofmann, D. Nashan, and L. Bruckner-Tuderman, "Petechiae on the fingertips as presenting symptom of dermatitis herpetiformis Duhring," Journal of the European Academy of Dermatology and Venereology, vol. 23, no. 6, pp. 732-733, 2009.

[24] S. Flann, C. Degiovanni, E. K. Derrick, and S. E. Munn, "Two cases of palmar petechiae as a presentation of dermatitis herpetiformis," Clinical and Experimental Dermatology, vol. 35, no. 2, pp. 206-208, 2010.
[25] J. Heinlin, B. Knoppke, E. Kohl, M. Landthaler, and S. Karrer, "Dermatitis herpetiformis presenting as digital petechiae," Pediatric Dermatology, vol. 29, no. 2, pp. 209-212, 2012.

[26] Y. Ohshima, Y. Tamada, Y. Matsumoto, and T. Hashimoto, "Dermatitis herpetiformis Duhring with palmoplantar keratosis," British Journal of Dermatology, vol. 149, no. 6, pp. 1300-1302, 2003.

[27] G. R. Powell, A. L. Bruckner, and W. L. Weston, "Dermatitis herpetiformis presenting as chronic urticaria," Pediatric Dermatology, vol. 21, no. 5, pp. 564-567, 2004.

[28] M. Saito, A. Böer, A. Ishiko, and T. Nishikawa, "Atypical dermatitis herpetiformis: a Japanese case that presented with initial lesions mimicking prurigo pigmentosa," Clinical and Experimental Dermatology, vol. 31, no. 2, pp. 290-291, 2006.

[29] M. Bowszyc-Dmochowska, A. Seraszek, E. Kaczmarek, J. Gornowicz, and M. Dmochowski, "Low strength of correlation between the intensity of neutrophil elastase expression in lesional skin and the level of serum IgA antibodies to epidermal transglutaminase in dermatitis herpetiformis," The Open Autoimmunity Journal. In press.

[30] S. J. P. Warren and C. J. Cockerell, "Characterization of a subgroup of patients with dermatitis herpetiformis with nonclassical histologic features," American Journal of Dermatopathology, vol. 24, no. 4, pp. 305-308, 2002.

[31] W. Dieterich, E. Laag, L. Bruckner-Tuderman et al., "Antibodies to tissue transglutaminase as serologic markers in patients with dermatitis herpetiformis," Journal of Investigative Dermatology, vol. 113, no. 1, pp. 133-136, 1999.

[32] P. Fabbri and M. Caproni, "Dermatitis herpetiformis," Orphanet Encyclopedia, 2005, http://www.orpha.net/data/ patho/GB/uk-DermatitisHerpetiformis.pdf.

[33] S. Kawana and A. Segawa, "Confocal laser scanning microscopic and immunoelectron microscopic studies of the anatomical distribution of fibrillar IgA deposits in dermatitis herpetiformis," Archives of Dermatology, vol. 129, no. 4, pp. 456-459, 1993.

[34] C. J. Ko, O. R. Colegio, J. E. Moss, and J. M. McNiff, "Fibrillar IgA deposition in dermatitis herpetiformis-an underreported pattern with potential clinical significance," Journal of Cutaneous Pathology, vol. 37, no. 4, pp. 475-477, 2010.

[35] S. E. Clements, C. M. Stefanato, B. Bhogal, and R. W. Groves, "Atypical dermatitis herpetiformis with fibrillar IgA deposition: CPC-8," British Journal of Dermatology, vol. 157, article 17, 2007.

[36] S. A. V. Jones, B. S. Bhogal, and M. M. Black, "Fibrillar IgA deposition may be associated with atypical dermatitis herpetiformis - a report of two cases," Journal of the European Academy of Dermatology and Venereology, vol. 7, no. 3, pp. 270-278, 1996.

[37] M. Shibahara, H. Nanko, M. Shimizu et al., "Dermatitis herpetiformis in Japan: an update," Dermatology, vol. 204, no. 1, pp. 37-42, 2002.

[38] D. Aeschlimann and V. Thomazy, "Protein crosslinking in assembly and remodelling of extracellular matrices: the role of transglutaminases," Connective Tissue Research, vol. 41, no. 1, pp. 1-27, 2000.

[39] J. Stamnaes, S. Dorum, B. Fleckenstein, D. Aeschlimann, and L. M. Sollid, "Gluten T cell epitope targeting by TG3 and TG6; implications for dermatitis herpetiformis and gluten ataxia," Amino Acids, vol. 39, no. 5, pp. 1183-1191, 2010.

[40] Ø. Molberg, S. N. Mcadam, R. Körner et al., “Tissue transglutaminase selectively modifies gliadin peptides that are 
recognized by gut-derived T cells in celiac disease," Nature Medicine, vol. 4, no. 6, pp. 713-717, 1998.

[41] Z. Nemes, L. Fésüs, A. Égerházi, A. Keszthelyi, and I. M. Degrell, " $\mathrm{N} \varepsilon$ ( $\gamma$-glutamyl)lysine in cerebrospinal fluid marks Alzheimer type and vascular dementia," Neurobiology of Aging, vol. 22, no. 3, pp. 403-406, 2001.

[42] T. M. Jeitner, M. B. Bogdanov, W. R. Matson et al., "N $\varepsilon-(\gamma-L-$ glutamyl)-L-lysine (GGEL) is increased in cerebrospinal fluid of patients with Huntington's disease," Journal of Neurochemistry, vol. 79, no. 5, pp. 1109-1112, 2001.

[43] A. Fasano and C. Catassi, "Current approaches to diagnosis and treatment of celiac disease: an evolving spectrum," Gastroenterology, vol. 120, no. 3, pp. 636-651, 2001.

[44] A. Rostom, J. A. Murray, and M. F. Kagnoff, "American gastroenterological association (AGA) institute technical review on the diagnosis and management of celiac disease," Gastroenterology, vol. 131, no. 6, pp. 1981-2002, 2006.

[45] A. M. Desai, R. S. Krishnan, and S. Hsu, "Medical Pearl: using tissue transglutaminase antibodies to diagnose dermatitis herpetiformis," Journal of the American Academy of Dermatology, vol. 53, no. 5, pp. 867-868, 2005.

[46] W. M. Porter, D. J. Unsworth, R. J. Lock, C. M. Hardman, B. S. Baker, and L. Fry, "Tissue transglutaminase antibodies in dermatitis herpetiformis," Gastroenterology, vol. 117, no. 3, pp. 749-750, 1999.

[47] I. Koop, R. Ilchmann, L. Izzi et al., "Detection of autoantibodies against tissue transglutaminase in patients with celiac disease and dermatitis herpetiformis," American Journal of Gastroenterology, vol. 95, no. 8, pp. 2009-2014, 2000.

[48] M. Caproni, C. Cardinali, D. Renzi, A. Calabró, and P. Fabbri, "Tissue transglutaminase antibody assessment in dermatitis herpetiformis," British Journal of Dermatology, vol. 144, no. 1, pp. 196-197, 2001.

[49] V. Kumar, M. Jarzabek-Chorzelska, J. Sulej, M. Rajadhyaksha, and S. Jablonska, "Tissue transglutaminase and endomysial antibodies-diagnostic markers of gluten-sensitive enteropathy in dermatitis herpetiformis," Clinical Immunology, vol. 98, no. 3, pp. 378-382, 2001.

[50] M. Sárdy, S. Kárpáti, B. Merkl, M. Paulsson, and N. Smyth, "Epidermal transglutaminase (TGase 3) is the autoantigen of dermatitis herpetiformis," Journal of Experimental Medicine, vol. 195, no. 6, pp. 747-757, 2002.

[51] T. D. Jaskowski, T. Hamblin, A. R. Wilson et al., "IgA antiepidermal transglutaminase antibodies in dermatitis herpetiformis and pediatric celiac disease," Journal of Investigative Dermatology, vol. 129, no. 11, pp. 2728-2730, 2009.

[52] M. F. Kagnoff, "AGA institute medical position statement on the diagnosis and management of celiac disease," Gastroenterology, vol. 131, no. 6, pp. 1977-1980, 2006.

[53] J. Alonso-llamazares, L. E. Gibson, and R. S. Rogers, "Clinical, pathologic, and immunopathologic features of dermatitis herpetiformis: review of the Mayo Clinic experience," International Journal of Dermatology, vol. 46, no. 9, pp. 910-919, 2007.

[54] E. Sugai, E. Smecuol, S. Niveloni et al., "Celiac disease serology in dermatitis herpetiformis. Which is the best option for detecting gluten sensitivity?" Acta Gastroenterologica Latinoamericana, vol. 36, no. 4, pp. 197-201, 2006.

[55] T. Matthias, S. Pfeiffer, C. Selmi, and M. E. Gershwin, "Diagnostic challenges in celiac disease and the role of the tissue transglutaminase-neo-epitope," Clinical Reviews in Allergy and Immunology, vol. 38, no. 2-3, pp. 298-301, 2010.

[56] T. D. Jaskowski, M. R. Donaldson, C. M. Hull et al., "Novel screening assay performance in pediatric celiac disease and adult dermatitis herpetiformis," Journal of Pediatric Gastroenterology and Nutrition, vol. 51, no. 1, pp. 19-23, 2010.

[57] M. Kasparkiewicz, C. Dähnrich, C. Probst et al., "Novel assay for detecting celiac disease-associated autoantibodies in dermatitis herpetiformis using deamidated gliadin-analogous fusion peptides," Journal of the American Academy of Dermatology, vol. 66, pp. 583-588, 2012. 


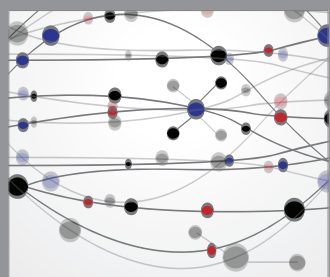

The Scientific World Journal
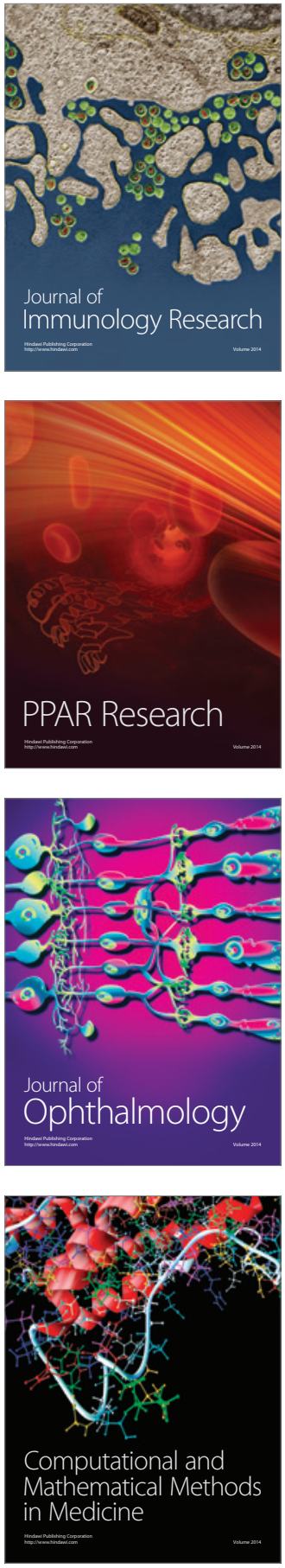

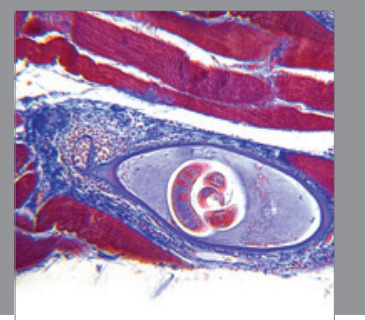

Gastroenterology

Research and Practice
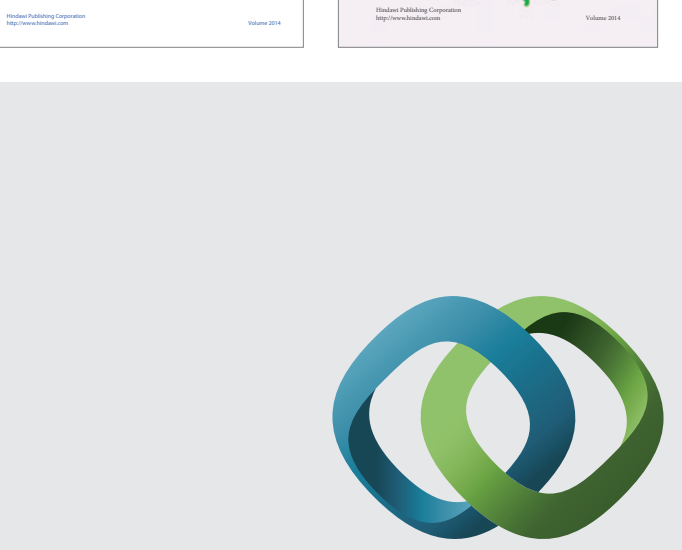

\section{Hindawi}

Submit your manuscripts at

http://www.hindawi.com
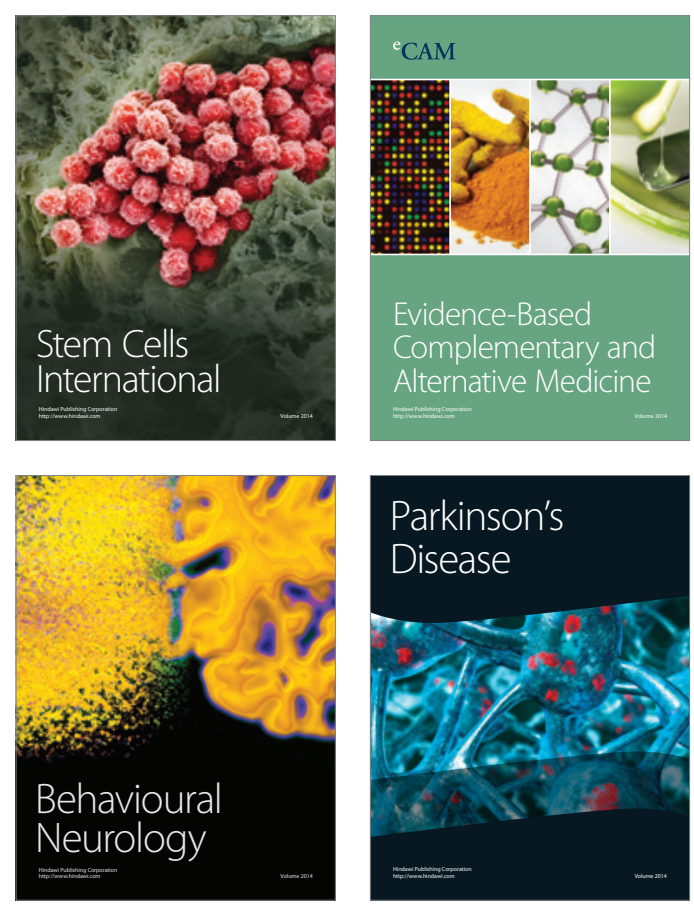

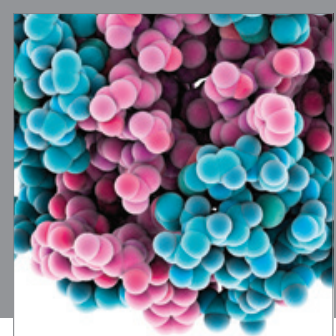

Journal of
Diabetes Research

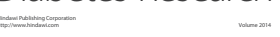

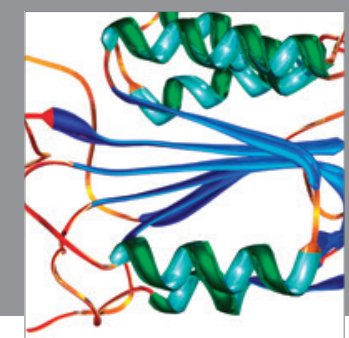

Disease Markers
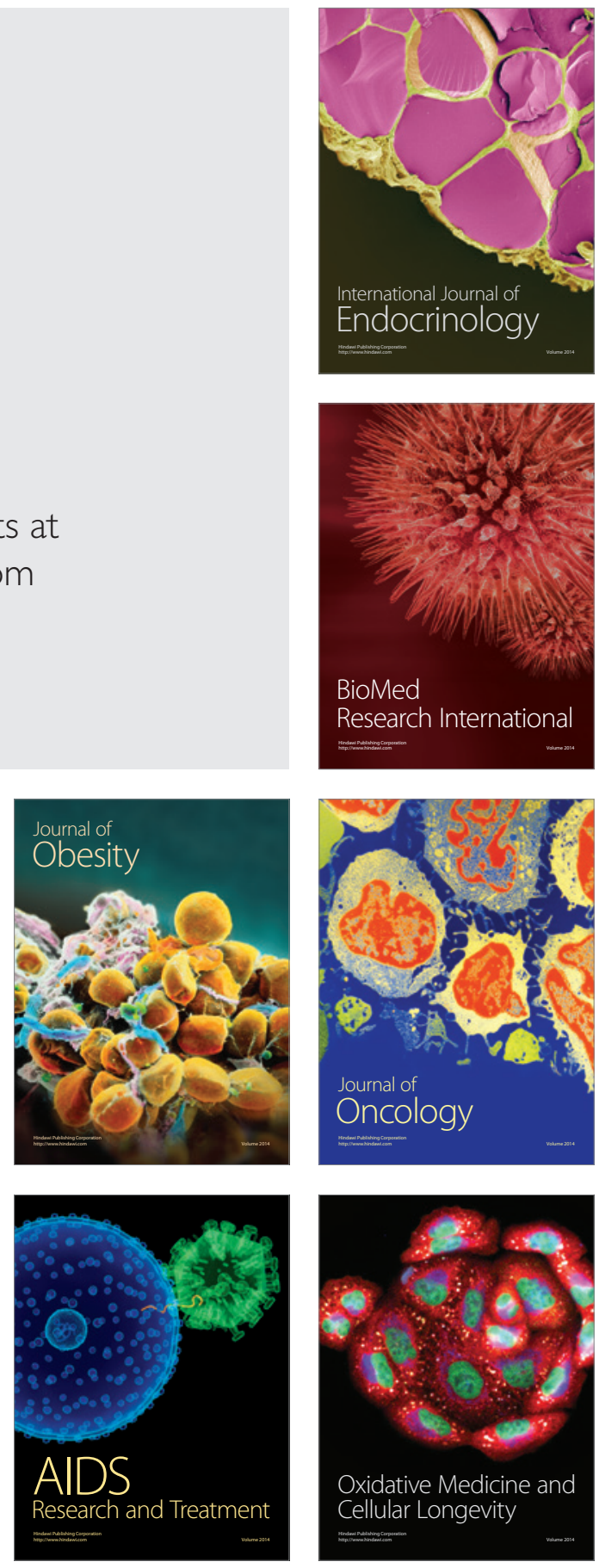